\title{
Increasing the level of network and information security using artificial intelligence
}

\author{
Roumen Trifonov $^{1}$, Georgi Tsochev ${ }^{2}$, Slavcho Manolov ${ }^{3}$, Radoslav Yoshinov ${ }^{4}$, Galya Pavlova ${ }^{5}$
}

\begin{abstract}
Too often, the unified security programs, based on comprehensive analyses of unified information from across the IT infrastructure, are costly, complex, difficult to implement and inefficient. As a result, most organizations lack accurate threat detection and informed risk-management capabilities. Therefore, the response to new information security threats can be a "security intelligence" approach. It is based on artificial intelligence and the use of its methods to protect from cybercrimes. The aim of this study is to present and compare different methods of artificial intelligence for fighting the crime in cyberspace, or rather their application in systems for detecting and preventing intrusions
\end{abstract}

Keywords-intrusion detection/prevention systems, artificial intelligence, computer networks, information security, intelligent methods, intelligent security

\section{Introduction}

It is very important for an organization is to develop mechanisms to secure to prevent unauthorized access to system resources and confidential company and government data [1]. There are many ways to protect the network infrastructure of an enterprise, including also systems for detecting and preventing intrusions. Over the past two decades, the development of computer networks is scope for innovative improvements. The market has many varieties of type "Next Generation", which provide a relatively good set of tools (systems) interaction and prevention of network attacks.

Physical devices such as sensors and detectors are not sufficient for monitoring, analysis and protection of the network infrastructure. It takes more complex information technology that can model proper behavior and identify abnormal. These systems cyber security need to be flexible, adaptable and clear, but at the same time able to discover the wide variety of threats and make smart choices.

With the pace of cyber-attacks, the human factor is not sufficient for timely analysis and action under attack. Human resources and lack of expertise were the main weakness of the organizations. The fact is that the intelligent agents carry out most network attacks, such as computer viruses and worms (fig. 1). So fighting them can become smart semi-autonomous or fully autonomous agents that can detect, evaluate and respond with appropriate action for protection. These intelligent methods will need to be able to manage the whole process in response to an attack, i.e. to analyze and determine what type of attack happens, what is intended and what is the appropriate countermeasures, and not least how to prioritize and secondary prevention of attacks. It was in those difficult situations we need innovative approaches by applying methods of artificial intelligence.
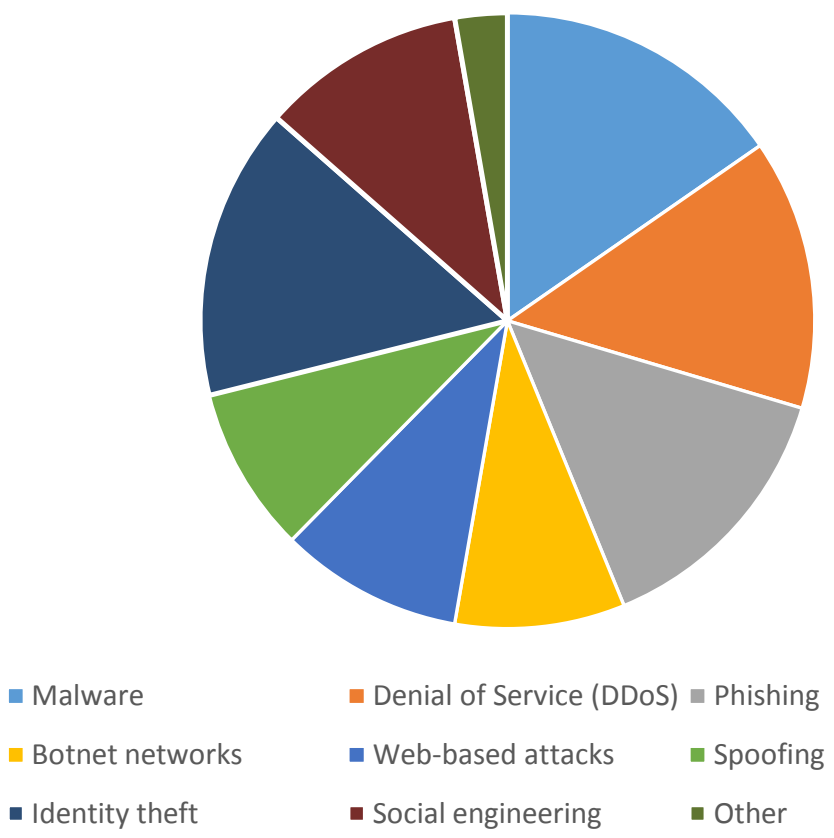

Figure 1Percentage of different attacks in 2016 
Physical devices such as sensors and detectors are not sufficient for monitoring, analysis and protection of the network infrastructure. It takes more complex information technology that can model proper behavior and identify abnormal. These systems cyber security need to be flexible, adaptable and clear, but at the same time able to discover the wide variety of threats and make smart choices.

With the pace of cyber-attacks, the human factor is not sufficient for timely analysis and action under attack. Human resources and lack of expertise were the main weakness of the organizations. The fact is that the intelligent agents carry out most network attacks, such as computer viruses and worms (fig. 1). So fighting them can become smart semi-autonomous or fully autonomous agents that can detect, evaluate and respond with appropriate action for protection. These intelligent methods will need to be able to manage the whole process in response to an attack, i.e. to analyze and determine what type of attack happens, what is intended and what is the appropriate countermeasures, and not least how to prioritize and secondary prevention of attacks. It was in those difficult situations we need innovative approaches by applying methods of artificial intelligence.

The aim of this study is to present and compare different methods of artificial intelligence for fighting the crime in cyberspace, or rather their application in systems for detecting and preventing intrusions.

\section{Artificial Intelligence}

As stated above, to provide a flexible and secure software that help people in fighting computer crime is necessary to use innovative technologies from artificial intelligence.

In recent years, artificial intelligence (AI) has become a tempting area for researchers. AI used to be breathed intelligence of a machine. The studies are highly technical and specialized; often fail to communicate with each other. Field of AI is based on the assertion that the intelligence of people (potential (innate) ability of a conscious individual to draw conclusions (deductions) on an information) can be described so precisely that a machine can simulate it. After several decades of research, AI is not only the subject of research or planning some movement, but also more complex depth and interrelated decisions.

AI offers many opportunities, most of which are inspired by nature computational methods - intelligent agents, neurons networks, data mining, artificial immune systems, machine learning, pattern recognition, fuzzy logic, heuristic and others. Applications in the field of AI are widely accepted by modern information society. This interdisciplinary initiative has created a joint connection between computer scientists and network engineers in the design, simulation and development of models for network penetration and their characteristics.

\section{Intrusion Detection/Prevention System}

Intrusion Detection/Prevention System (abbreviated as IDPS) is a security system that detects hostile activity on the network and tries to prevent it. The key is then to detect and possibly prevent actions that could jeopardize the security of the system, or attempt to break in the work, including the phases of exploration / collection of data that include, for example, a port scan. One of the key features of intrusion detection/prevention systems is their ability to provide a view of the unusual activity and issue alarms notifies administrators and / or block the connection of the suspect [2].

The classification of the typical IDPS systems is divided into three large groups [4] is given in table 1.

IDPS consist of four major elements (fig. 2) - data collection, feature selections, analysis and action.

The data collection is a file in which is recorded the data that should be analyzed. In rule based IDPS the analysis is done by checking the data of compare it to a signature or pattern. Another method is anomaly based. The action defines the attack and reaction of the system [5]. Usually the information flow in IDPS starts with the row packet capture this involves not only capturing packets, but also passing the data to the next component of the system.

TABLE I. INTRUSION DETECTION/PREVENTION TYPES

\begin{tabular}{|c|c|}
\hline Name & Functionality \\
\hline $\begin{array}{c}\text { Host Based Intrusion } \\
\text { Setection/Prevention } \\
\text { System }\end{array}$ & $\begin{array}{c}\text { collecting information } \\
\text { about activity on a } \\
\text { particular single } \\
\text { system or host }\end{array}$ \\
\hline $\begin{array}{c}\text { Network Based Intrusion } \\
\text { Detection/Prevention } \\
\text { System }\end{array}$ & $\begin{array}{c}\text { perform analysis of all } \\
\text { traffic passing through } \\
\text { a network segment or } \\
\text { subnet }\end{array}$ \\
$\begin{array}{c}\text { Application Based } \\
\text { Intrusion }\end{array}$ & $\begin{array}{c}\text { focuses its monitoring } \\
\text { and analysis on a } \\
\text { specific application } \\
\text { protocol or protocols } \\
\text { in use by the } \\
\text { computing system }\end{array}$ \\
\hline
\end{tabular}

Filtering means limiting the packets that are captured according to a certain logic based on characteristics, such as type of packet, IP source address range, and others. Subsequently sending the packets to a series of decoder routines that define the packet structure for the layer two data that are collected through promiscuous monitoring. Once each packet is decoded, it is often stored either by saving its data to a file or by assimilating it into a data structure while, at the same time, the data are cleared from memory. Decoding 
"makes sense" out of packets, but this, in and of itself, does not solve all the problems that need to be solved for an IDPS to process the packets properly. Stream reassembly means taking the data from each TCP stream and, if necessary, reordering it [6].

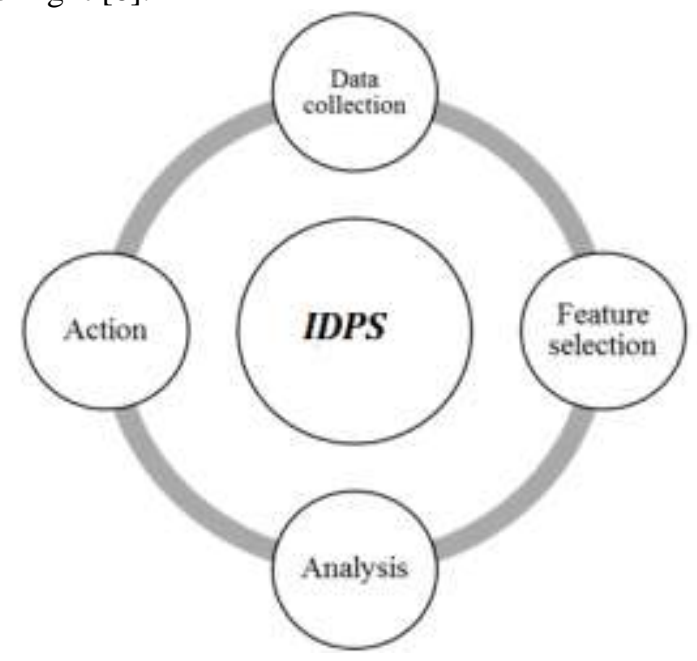

Figure 2 Functionality of IDPS

The typical components in an IDPS are sensor or agent, management server, database server and console [5]. The role of the artificial intelligence is the development of the sensors or agents. Typically using the term sensor is for IDPSs that monitor networks, including network-based, wireless, and network behavior analysis technologies. In host-based IDPS technologies, the term agent is used. Some IDPS sensors have a learning or simulation mode that suppresses all prevention actions and instead indicates when a prevention action would have been performed [5].

Artificial intelligence easily solve the problem of human factor. The primary difficulty of the IDPS is how accurately the system can in terms of whether non-hostile activity is flagged; false positive and whether malicious activity will be missed; false negative. Large volume of alerts is unmanageable and overwhelming to the human analyst. Inspecting thousands of alerts per day is unfeasible, especially if $99 \%$ of them are false alerts. Many approaches have been suggested, but artificial intelligence comes natural to solve this problem - machine learning, data mining etc.

\section{Using artificial intelligence to protect against cybercrime}

An intelligent, adaptive and cost-effective tool that is capable of detecting and preventing intrusions in real time is the purpose companies that deal with cybercrime. Various methods in the field of AI has been used to automate the process of detecting intrusions while reducing human factor.

\section{A. Artificial Neural networks (ANN)}

An Artificial Neural Network is an information processing system that is inspired by the way biological nervous systems.
Neural networks are models built from multiple processing elements (neurons), each of which perform simple numerical operations and share results with their neighbors through weighted connections. Most of the intrusion detection systems based on the ANN are using two kinds of neural networks: multilayered feedforward neural networks and Kohonen's selforganizing maps [7].

ALAN BIVENS et all [8] developed a system that uses use self-organizing maps, as they have been shown to be effective in novelty detection, automated clustering and visual organization. Their system is modular based network IDS that analyses the tcpdump traffic and develop windowed traffic intensity trends. The learning process uses architectural learning period.

Dilip Kumar Barman and Guruprasad Khataniar [9] (figure 4) used Artificial Neural Network (ANN) with back propagation as IDPS. The ANN based IDPS system will use the attributes (total of 41) of the intrusion signatures of the dataset KDD99.

Min-Joo Kang and Je-Won Kang proposed a novel IDS using a deep neural network (DNN) is proposed to enhance the security of in-vehicular network [10].

Mehdi MORADI and Mohammad ZULKERNINE [11] present A Multi-Layer Perceptron (MLP) is used for intrusion detection based on an off-line analysis approach. Their research aims to solve a multi class problem in which by the neural network the type of detected attack.

\section{B. Artificial Immune Systems}

The Artificial Immune Systems (AIS) were inspired by the Human Immune System that is robust, decentralized, error tolerant, and adaptive [12]. The HIS is made of molecules, cells, and tissues that establish human body's resistance to infections caused by viruses and etc. The AIS can distinguish and eliminate the different pathogens from self-cells. This provides a great source of inspiration for the security of computer systems, especially IDS.

The first who began researches in this field are Farmer, Packard, and Perelson. Their algorithm describes a method for change detection that is based on the generation of T-cells in the immune system. In 1994 Forrest and her group at the University of New Mexico began research to build an IDS based on AIS. They proposed a negative selection algorithm to utilize the process of the HIS for a sophisticated anomalydetection process [13].

Liu et al. propose method of intrusion detection for the IoT that simulates self and non-self-antigen. The Immature detector, mature detector and the memory detector evolve dynamically to prevent intrusion. Their algorithm provides a new way in the in the intrusion detection in IoT environment [14].

\section{Machine learning}

Machine learning methodologies are being widely used by the researchers in the field of network intrusion detection due 
to their generalization capabilities that helps to understand the technical knowledge about the intrusions that do not have any predefined patterns [16]. There are two types of machine learning techniques - single classifier and hybrid classifier. Juma et. al. [17] are very well described the machine learning techniques in their paper. They say that the future of machine learning in intrusion detection prevention systems, are only beginning to develop and can be expected many more future scientific developments.

\section{Fuzzy logic and fuzzy sets}

Fuzzy set theory was introduced by Zadeh [18] for handling uncertainty.

Fuzzy logic is a rule-based system that can rely on the practical experience of an operator, particularly useful to capture experienced operator knowledge [19]. The approach of FL imitates the way of decision making in humans that involves all intermediate possibilities ranges in degree between 0 and 1 .

Jongsuebsuk et al. [20] proposed a network IDS based on a fuzzy genetic algorithm. Fussy rules are used to classify network attack data, while a genetic algorithm is an optimization algorithm that can help finding appropriate fuzzy rule and give the best/optimal solution.

Chimphlee et al. proposed the Fuzzy Rough C-means (FRCM) to clustering analysis [21]. The results they achieve with the performance are very good compared to the Kmeans methods.

\section{E. Genetic Algorithms}

Genetic Algorithms incorporate the concept of Darwin's theory. They were inspired by the biological evolution (development), natural selection, and genetic recombination [12]. Genetic algorithms can be used to evolve simple rules for network traffic. GA generates a set of rules, that later can be used to distinguish the normal and abnormal network traffic. The algorithms to create these data sets uses a chromosomelike data structure and evolve the chromosomes using selection, recombination and mutation operators.

Li [22] proposed an IDS with 57 genes in chromosomes, where each gene represents single connection feature, like: source IP address, destination IP address, source port, destination port, duration, protocol, number of bytes sent by originator, number of bytes sent by responder, etc. Due to the effectiveness of the evaluation function, the succeeding populations are biased toward rules that match intrusive connection.

Anup Goyal And Chetan Kumar [23],suggested systematic learning method known as Genetic Algorithm (GA),to identify illegitimate nodes. The algorithm considers the varied features in network connectivity like protocol type, network service to destination and connection status to generate a type based rules

Ojugo, et. al. have used genetic algorithms to develop rulebased intrusion detection. their study, the genetic algorithm based approach, which uses a set of classification rules derived from the data network audit and support confidence framework to be used as a fitness function, to evaluate the quality of each rule. Implementation of the software aims to improve system security in the network settings to allow the confidentiality, integrity and availability of system resources [24].

Immannavar et al. proposed A GNP based fuzzy membership for identifying threats, attacks or intrusions over the Internet [25]. There methods handle both discrete and continuous attributes and it can be flexibly applied to any kind of attacks.

\section{F. Intelligent agents}

Agents can be defined to be autonomous, problem-solving computational entities capable of effective operation in dynamic and open environments [26]. Agents are often deployed in environments in which they interact, and may be cooperate, with other agents (including both people and software) that have possibly conflicting aims.

Ganpathy et al. in 2012 proposed a method that combines an intelligent agent-based weighted distance outlier detection (IAWDBOD) algorithm and intelligent agent-based enhanced multiclass support vector Machine (IAEMSVM) algorithm. The result for DoS, Probe, and other attacks are $99.77 \%$, $99.70 \%$, and $79.72 \%$, respectively, when intelligent agents are added to the classifier. The main advantage of this method is that it reduces the false positive rates [27].

Jain et al. in their article make detailed comparative analysis of different mobile agent based IDS [28].

\section{v. Comparison of the different AI methods}

AI have many advantages such as - precision and accuracy, fraud detection, lacking the emotional side, robots think logically, function without stopping (do not require sleep or breaks), the costs are minimized and controlled and etc. Of course, like any fast growing technology there are some problems - cannot act any different from what they are programmed to do, machines lack of common sense, eventually will replace humans in every field and will lead to unemployment, fear of robots superseding humans etc.

Several algorithms for intrusion detection and prevention based on various methods were reviewed and the advantages and disadvantages of these algorithms are shown in table 2.

\section{vI. Conclusion}

AI gives us the opportunity to develop autonomous computing solutions that adapt to their context of use, using the methods of self-control, self-tuning and self-configuration, self-diagnostics and self-healing. When it comes to the future of information security, AI looks very promising area of research that focuses on improving the security of cyberspace.

This article looks at some of the areas of AI, which have undergone significant changes over the last decade. It shows the progress that scientists have made in the fight against cybercrime. 
TABLE II. ADVANTAGES AND DISADVANTAGES OF THE DIFFERENT AI METHODS USED FOR IDPS

\begin{tabular}{|c|c|c|}
\hline Technology & Advantages & Disadvantages \\
\hline $\begin{array}{l}\text { Artificial } \\
\text { Neural } \\
\text { Networks }\end{array}$ & $\begin{array}{l}\text { learn by example or training, } \\
\text { flexibility; } \\
\text { multiple class classification; } \\
\text { Parallel nature; } \\
\text { able to work imprecise and incomplete data; } \\
\text { speed; } \\
\text { ability to "learn" the characteristics of } \\
\text { misuse attacks and identify instances that are unlike } \\
\text { any which have been observed before by the network [29] }\end{array}$ & $\begin{array}{l}\text { Indication of intrusions completely } \\
\text { dependents on the training of the system } \\
\text { and the the training data; } \\
\text { training routine requires a large number } \\
\text { of data to ensure that the results are } \\
\text { statistically accurate. }\end{array}$ \\
\hline $\begin{array}{l}\text { Artificial } \\
\text { Immune } \\
\text { Systems }\end{array}$ & $\begin{array}{l}\text { Decentralized; } \\
\text { error tolerant; } \\
\text { adaptive; } \\
\text { highly efficient and versatile robustness } \\
\text { distribution } \\
\text { lightweight } \\
\text { self-organizing, and self-adapting }\end{array}$ & $\begin{array}{l}\text { Work well on small problems with } \\
\text { medium sized testbeds; } \\
\text { Hard implementation for distinguishing } \\
\text { self from non-self pathogens }\end{array}$ \\
\hline $\begin{array}{l}\text { Machine } \\
\text { learning }\end{array}$ & $\begin{array}{l}\text { High Accuracy } \\
\text { Able to model complex and nonlinear decision boundaries. }\end{array}$ & $\begin{array}{l}\text { Difficulties with evaluation; } \\
\text { Outlier detection }\end{array}$ \\
\hline $\begin{array}{l}\text { Fuzzy } \\
\text { Logic/Sets }\end{array}$ & $\begin{array}{l}\text { Reasoning is Approximate rather than precise; } \\
\text { Effective (port scans and probes) } \\
\text { Reconciles conflicting objectives } \\
\text { fuzzy sets easily modified }\end{array}$ & $\begin{array}{l}\text { High consumption of resources; } \\
\text { Difficulty in relevant rule subset } \\
\text { identification; }\end{array}$ \\
\hline $\begin{array}{l}\text { Genetic } \\
\text { Algorithms }\end{array}$ & $\begin{array}{l}\text { deriving best classification rules; } \\
\text { Selecting optimal parameters } \\
\text { solves the problems with multiple solutions } \\
\text { intrinsic parallelism; } \\
\text { highly re-trainable } \\
\text { evolve over time by using crossover and mutation [30] }\end{array}$ & $\begin{array}{l}\text { Pver-fitting; } \\
\text { Complex representing of the problem; } \\
\text { Complex configuration of the system; } \\
\text { Converge premature to a solution; }\end{array}$ \\
\hline $\begin{array}{l}\text { Intelligent } \\
\text { Agents }\end{array}$ & $\begin{array}{l}\text { Mobility } \\
\text { Adaptability } \\
\text { Collaboration } \\
\text { Autonomy agents are independently-running entities } \\
\text { Inferential capability } \\
\text { Pro-activeness: agents can take the initiative to act and response to } \\
\text { their environment } \\
\text { low cost and time saving approach } \\
\text { Reducing Network Load } \\
\text { Platform Independence [31] }\end{array}$ & $\begin{array}{l}\text { The scalability is limited because the } \\
\text { analysis is performed in one single. } \\
\text { Hard to maintenance and control } \\
\text { overhead; } \\
\text { Tool are new and have unknown security } \\
\text { bugs and vulnerabilities; } \\
\text { Agents ability to travel introduces fault- } \\
\text { tolerant properties [32] }\end{array}$ \\
\hline
\end{tabular}

This area is fast growing and requires in-depth research, as there are many promising results that may be obtained from these algorithms, especially in their combined use.

Also the combination of Network and Host-based IDPS (NIDPS, HIDPS) in a fully distributed framework structure in a networking environment with $\mathrm{AI}$ is readily applicable to any network and its requirements and the results are encouraging, but it is still too early to declare any definitive conclusions

\section{Acknowledgment}

This research is conducted and funded in relation to the execution of a scientific-research project № H07/56 "Increasing the level of network and information security using intelligent methods" under the contract with National Science Fund in Bulgaria.

\section{References}

[1] Roumen Trifonov, R. Yoshinov, "Some Security Issues of the Governmental Cloud," in 15th International Conference on ACE'16, Mallorca, Spain, August 19-21, 2016.

[2] P. Kazienko and P. Dorosz, "Intrusion Detection Systems (IDS) Part I (network intrusions; attack symptoms; IDS tasks; and IDS architecture)," 7 April 2003. http://www.windowsecurity.com/articlestutorials/intrusion_detection/Intrusion_Detection_Systems_IDS_Part_I_ _network_intrusions_attack_symptoms_IDS_tasks_and_IDS_architectur e.html. 
[3] D. Stiawan, A. H. Abdullah and M. . Y. Idris, "Characterizing Network Intrusion Prevention System," International Journal of Computer Applications, vol. 14, no. 1, 2011.

[4] "Host- vs. Network-Based Intrusion Detection Systems," SANS Institute 2000 - 2005, 2016.

[5] K. Scarfone and P. Mell , Guide to Intrusion Detection and Prevention Systems (IDPS), National Institute of Standards and Technology, 207.

[6] Y. Kumar and S. Dhawan, "A Review on Information Flow in Intrusion Detection System," IJCEM International Journal of Computational Engineering \& Management, vol. 15, no. 1, pp. 91-96, 2012.

[7] A. Vesely and D. Brechlerova, "Neural networks in intrusion detection systems," Agric. econ. - Czech, vol. 50, pp. 35-39, 2004.

[8] A. Bivens, C. Palagiri, R. Smith, B. Szymanski and M. Embrechts, "Network-Based Intrusion Detection Using Neural," in Intelligent Engineering Systems through Artificial Neural Networks ANNIE-2002, St. Louis, 2002.

[9] D. K. Barman and G. Khataniar, "Design Of Intrusion Detection System Based On Artificial Neural Network And Application Of Rough Set," International Journal of Computer Science \& Communication Networks, vol. 2, no. 4, pp. 549-552.

[10] M.-J. Kang and J.-W. Kang, "Intrusion Detection System Using Deep Neural Network for In-Vehicle Network Security," PLOS one, 7 Jun 2016. https://www.ncbi.nlm.nih.gov/pmc/articles/PMC4896428/.

[11] M. Moradi and M. Zulkernine, "A Neural Network Based System for Intrusion Detection and Classification of Attacks," Queen University, Canada, 2004

[12] A. S. A. Aziz, M. Salama and A. e. Hassanien, "Detectors Generation using Genetic Algorithm for a Negative Selection Inspired Anomaly Network Intrusion Detection System," in Federated Conference on Computer Science and Information Systems, WROCŁAW, 2012.

[13] S. Forrest, S. A. Hofmeyr and A. Somayaji, "Computer Immunology," Commun. ACM, vol. 40, no. 10, p. 88-96, 1997.

[14] C. Liu, J. Yang, Y. Zhang, R. Chen and J. Zeng, "Research on immunity-based intrusion detection technology for the Internet of Things," Seventh International Conference on Natural Computation, 2011.

[15] I. Dutt, S. Borah and I. Maitra, "Intrusion Detection System using Artificial Immune System," International Journal of Computer Applications, vol. 144, no. 12, pp. 19-22, 2016.

[16] M. Panda, A. Abraham, S. Das and M. R. Patra, "Network Intrusion Detection System: A Machine Learning Approach," Intelligent Decision Technologies, vol. 5, no. 4, 2011.

[17] S. Juma, Z. Muda, M. A. Mohamed and W. Yassin , "Machine Learning Techniques for Intrusion Detection System: A Review," Journal of Theoretical and Applied Information Technology, vol. 72, no. 3, pp. 422-429, 2015.

[18] S. RAJASEKARAN and G. A. VIJAYALAKSHMI PAI, Neural Networks, Fuzzy Logic and Genetic Algorithm: Synthesis and Applications, PHI Learning Pvt. Ltd., 2003.

[19] N. Dingle, "Artificial Intelligence: Fuzzy Logic Explained," http://www.controleng.com/single-article/artificial-intelligence-fuzzylogic-explained/8f3478c13384a2771ddb7e93a2b6243d.html.

[20] P. Jongsuebsuk, N. Wattanapongsakorn and C. Charnsripinyo, "Realtime intrusion detection with fuzzy genetic algorithm," 10th International Conference on Electrical Engineering/Electronics, Computer, Telecommunications and Information Technology, 2013.

[21] W. Chimphlee, A. H. Abdullah and M. N. M. Sap, "Anomaly-Based Intrusion Detection using Fuzzy Rough Clustering," International Conference on Hybrid Information Technology, 2006.

[22] W. Li, "Using Genetic Algorithm for Network Intrusion Detection," Proc. of the United States Department of Energy Cyber Security Group, 2004

[23] A. Goyal and C. Kumar, "GA-NIDS: A Genetic Algorithm based Network Intrusion Detection System," 2007.

[24] A. Ojugo, A. Eboka, O. Okonta, R. Yoro and F. Aghware, "Genetic Algorithm Rule-Based Intrusion Detection System (GAIDS)," Journal of Emerging Trends in Computing and Information Sciences, vol. 3, no. 8, pp. 1182-1194, 2012.
[25] M. Immannavar, P. M. Pujar and M. Suryavanshi , "An Intrusion Detection Model Based on Fuzzy Membership Function Using GNP," International Journal of Research in Engineering and Technology, vol. 4, No. 8, pp. 27-32, 2015.

[26] M. Luck, P. McBurney and C. Preist, Agent Technology: Next Generation Computing, AgentLink II, 2003.

[27] S. Ganapathy, P. Yogesh and A. Kannan, "Intelligent Agent-Based Intrusion Detection System Using Enhanced Multiclass SVM," Computational Intelligence and Neuroscience, 2012.

[28] C. Jain and A. K. Saxena, "General Study of Mobile Agent Based Intrusion Detection System (IDS)," Journal of Computer and Communications, vol. 4, pp. 93-98, 2016.

[29] K. S. Devikrishna and B. B. Ramakrishna , "An Artificial Neural Network based Intrusion Detection System and Classification of Attacks," International Journal of Engineering Research and Applications (IJERA), vol. 3, no. 4, pp. 1959-1964, 2013.

[30] P. G. Majeed and S. Kumar, "Genetic Algorithms in Intrusion Detection Systems: A Survey," International Journal of Innovation and Applied Studies, vol. 5, no. 3, pp. 233-240, 2014.

[31] H. Albag, "Network \& Agent Based Intrusion Detection Systems," https://www7.informatik.tumuenchen.de/um/courses/seminar/worm/WS0405/albag.pdf.

[32] T. Karygiannis, "Network Security Testing Using Mobile Agents," National Institute of Standards and Technology, Gaithersburg,.

About Author (s):

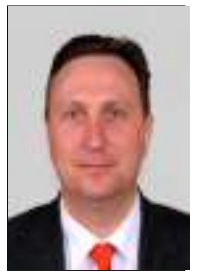

1. Assoc.Prof. R.Trifonv is a lecturer at the Technical University of Sofia. He is a head of department "Information Technologies in Industry. His fields of research are artificial intelligence, systems and information security, e-government.

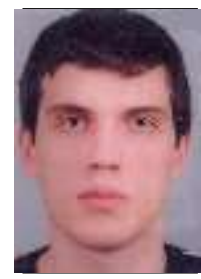

2. Mag. Eng. G. Tsochev is a Ph.D. student at the Technical University of Sofia and has experience as system and network administrator. His fields of research are network and information security, intelligent angents.

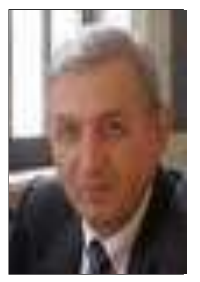

3. Assoc. Prof. S. Manolov works at the Technical University of Sofia. He has experience and research publications in the field of: Electronic Governance; System Integration and Interoperability; Network and Information Security

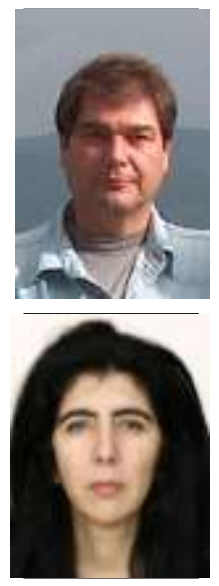

4. Assoc.Prof. R. Yoshinov is Director of Laboratory of Telematics at the Bulgarian Academy of Sciences. Research in the field of telecommunications, computer networks, Modelling and creation of heterogeneous, distributed network education environments for e-learning.

5. Assist.Prof. G. Pavlova is a PhD student at the at the Technical University of Sofia. Her fields of research application of artificial intelligence methods in robotics, machine learning and data mining, information security. 